\title{
Pasado, presente y futuro del tratamiento conservador del cáncer de cabeza y cuello
}

\section{Sr. Director}

En respuesta al editorial publicado recientemente en esta revista Pasado, presente y futuro del tratamiento conservador del cáncer de cabeza y cuello, firmado por el Dr. A. Villar Rodríguez', nos gustaría hacer algunas consideraciones sobre ciertas afirmaciones contenidos en él.

Se hace en el editorial una defensa de los estudios piloto encadenados como la forma mejor y más rápida de avanzar en el conocimiento de las mejores técnicas para el tratamiento del cáncer de cabeza y cueIlo, por encima de los estudios randomizados que, según deja entender el autor, enlentencen el desarrollo científico y hacen que las técnicas actuales de quimioterapia y radioterapia concurrente estén en una fase de semiempirismo. Es bien conocido y aceptado por toda la comunidad científica, que el mejor método para avanzar en el conocimiento médico y abandonar cualquier tipo de actitud empírica es seguir el método científico. Es verdad que dicho método es lento pero garantiza evitar poner a los pacientes en riesgo de recibir tratamientos que aunque puedan parecer superiores en estudios piloto o estudios fase II, al llegar el momento de ser comparados (estudios randomizados) pueden resultar no ser mejores que el tratamiento estándar o incluso demostrar ser inferiores. Un ejemplo muy reciente se ha vivido con la utilización de la eritropoyetina como tratamiento de soporte de la anemia inducida por los tratamientos oncológicos. Se conoce el impacto negativo de la anemia y la hipoxia en la supervivencia de los pacientes con cáncer de cabeza y cuello. Desde muchos foros y desde muchos estudios piloto, se ha defendido la utilización de eritropoyetina para la corrección de la anemia en pacientes con cáncer. Se decía que si eritropoyetina era capaz de corregir la anemia, de forma analógica esta corrección mejoraría la supervivencia de los pacientes tratados. Pues bien, el primer estudio randomizado en pacientes con cáncer de cabeza y cuello cuyo objetivo principal es la medición del impacto de eritropoyetina en la supervivencia, demuestra una peor supervivencia de los pacientes en la rama de eritropoyetina en relación con la rama de tratamiento con radioterapia sola ${ }^{2}$. Este estudio randomizado, a pesar de todas las críticas que ha recibido, ha supuesto una piedra de toque para el uso de eritropoyetina en pacientes en curso de tratamiento radical. En estos momentos no queda claro la seguridad de utilizar eritropoyetina en el tratamiento radical de estos tumores. Utilizar sólo los estudios piloto para avanzar en el conocimiento científico puede suponer un riesgo para el tratamiento de nuestros pacientes, pero además puede suponer un retraso en el avance científico ya que se estarían reclutando pacientes para estudios que no permiten, al final, sacar ninguna conclusión definitiva.

El avance en los conocimientos de quimioterapia y radioterapia concomitante es sólido y seguro. Existen en estos momentos 2 meta-análisis ${ }^{3,4}$ y al menos 8 estudios randomizados ${ }^{5-12}$ que demuestran un incremento de un $15-20 \%$ de supervivencia del tratamiento concomitante con relación a la radioterapia sola. Ninguna otra forma de tratamiento en cáncer de cabeza y cuello ha demostrado jamás dichos incrementos de supervivencia. En estos momentos, oncólogos médicos y radioterapeutas de todo el mundo deberíamos diseñar estudios encaminados a responder cuáles son las mejores combinaciones en cada una de las localizaciones tumorales. Sólo mediante estudios randomizados podremos responder a los retos que se nos presentan.

\footnotetext{
R. Mesía Nin*, M. García Martín**, J. R. Germá Lluch*** * Responsable de Cáncer de Cabeza y Cuello Servicio de Oncología Médica ** Coordinador de Investigación Clínica Institut Català d'Oncologia. L'Hospitalet, Barcelona *** Jefe de Servicio de Oncología Médica Institut Català d'Oncologia Hospital Durán i Reynals. L'Hospitalet, Barcelona
} 


\section{Bibliografía}

1. Villar Rodríguez A. Pasado, presente y futuro del tratamiento conservador del cáncer de cabeza y cuello. Oncología 2004; 27(1):1-3.

2. Henke $M$, Laszig R, Rübe $C$ y col. Erythropoletin to treat head and neck cancer patients with anaemia undergoing radiotherapy: randomised, double-blind, placebocontrolled trial. Lancet 2003; 362:1255-60.

3. El-Sayed S, Nelson N. Adjuvant and adjunctive chemotherapy in the management of squamous cell carcinoma of the head and neck region: a meta-analysis of prospective and randomized triais. J Clin Oncol 1996; 14:838-47.

4. Pignon JP, Bourhis J, Domenge C, Designé L. Chemotherapy added to locoregional treatment for head and neck squamous-cell carcinoma: three meta-analyses of updated individual data. MACH-NC Collaborative Group. Meta-Analysis of Chemotherapy on Head and Neck Cancer. Lancet 2000; 355:949-55 .

5. Merlano M, Benasso M, Corvo R y col. Five-year update of a randomized trial of alternating radiotherapy and chemotherapy compared with radiotherapy alone in treatment of unresectable squamous cell carcinoma of the head and neck. J Natl Cancer Inst 1996; 88:583-9.

6. Brizel DM, Albers MA, Fisher SR y col. Hyperfractionated irradiation with or without concurrent chemotherapy for locally advanced head and neck cancer. N Engl J Med 1998; 338:1798-804.

7. Wendt TG. Grabenbauer GG, Rodel CM y col. Simult- neous radiochemotherapy versus radiotherapy alone in advanced head and neck cancer: A randomized multicenter study. J Clin Oncol 1998; 16:1318-24.

8. Calais G, Alfonsi M, Bardet E y col. Randomized study comparing radiation alone versus radiotherapy with concomitant chemotherapy in stages lil and IV oropharynx carcinoma. Preliminary results of the 94.01 study from the French group of radiation oncology for head and neck cancer. J Nat Cancer Inst 1999; 91 (24):2082-6.

9. Jeremic B, Shibato Y, Milicic B y col. Hyperfractionated radiation therapy with or without concurrent low-dose doily cisplatin in locally advanced squamous cell carcinoma of the head and neck. A prospective randomized trial. J Clin Oncol 2000; 18(7): 1458-64.

10. Adelstein DJ, Li Y, Adams GL y col. An intergroup phase lil comparison of standard radiation therapy and two scheduies of concurrent chemoradiotherapy in patients with unresectable squemous cell chead and neck cancer. J Clin Oncol 2003; 21(1):92-8.

11. Al-Sarraf M, LeBlanc M, Shanker PG y col. Chemoradiotherapy versus radiotherpy in patients with advanced nasopharyngeal cancer. Phase lil randomized intergroup study 0099. J Clin Oncol 1998; 16:1310-7.

12. Lin JC, Jan JS, Hsu CY, Liang WM, Jiang RS, Wang WY. Phase lil study of concurrent chemoradiotherapy versus radiotherapy alone for advanced nasopharyngeal carcinoma: positive effect on overall and progressionfree survival. J Clin Oncol. 2003; 21(4):631-7. 